\title{
Efficacy of programmed intermittent bolus epidural analgesia in thoracic surgery: a randomized controlled trial
}

\author{
M. Higashi ${ }^{1}$, K. Shigematsu², E. Nakamori ${ }^{1}$, S. Sakurai ${ }^{1}$ and K. Yamaura ${ }^{1 *}$ (id
}

\begin{abstract}
Background: Continuous epidural infusion (CEI) has some disadvantages, such as increased local anesthetic consumption and limited area of anesthetic distribution. Programmed intermittent bolus (PIB) is a technique of epidural anesthesia in which boluses of local anesthetic are automatically injected into the epidural space. The usefulness of PIB in thoracic surgery remains unclear. In this study, we aimed to compare the efficacies of PIB epidural analgesia and CEl in patients undergoing thoracic surgery.
\end{abstract}

Methods: This randomized prospective study was approved by the Institutional Review Board. The study included 42 patients, who were divided into CEI $(n=21)$ and PIB groups $(n=21)$. In the CEI group, patients received continuous infusion of the local anesthetic at a rate of $5.1 \mathrm{~mL} / 90 \mathrm{~min}$. In the PIB group, a pump delivered the local anesthetic at a dose of $5.1 \mathrm{~mL}$ every $90 \mathrm{~min}$. The primary endpoints were the frequency of patient-controlled analgesia (PCA) and the total dose of local anesthetic until $36 \mathrm{~h}$ following surgery. Student's $t$-test, the chi-square test, and the Mann-Whitney $U$ test were used for statistical analyses.

Results: The mean number of PCA administrations and total amount of local anesthetic were not significantly different between the two groups up to $24 \mathrm{~h}$ following surgery. However, the mean number of PCA administrations and total amount of local anesthetic at 24-36 h after surgery were significantly lower in the PIB group than in the CEl group (median [lower-upper quartiles]: 0 [0-2.5] vs. 2 [0.5-5], $P=0.018$ and 41 [41-48.5] vs. 47 [43-56], $P=0.035$, respectively). Hypotension was significantly more frequent in the PIB group than in the CEI group at $0-12 \mathrm{~h}$ and $12-24 \mathrm{~h}$ ( $3.3 \%$ vs. $0.5 \%, P=0.018$ and $7.9 \%$ vs. $0 \%, P=0.017$, respectively).

Conclusion: PIB can reduce local anesthetic consumption in thoracic surgery. However, it might result in adverse events, such as hypotension.

Trial registration: This randomized prospective study was approved by the Institutional Review Board (IRB No. 15-9-06) of the Fukuoka University Hospital, Fukuoka, Japan, and was registered in the clinical trials database UMIN (ID 000019904) on 24 November 2015. Written informed consent was obtained from all patients.

Keywords: Anesthesia, Epidural anesthesia, Programmed intermittent bolus, Thoracic surgery

\footnotetext{
* Correspondence: keny@kuaccm.med.kyushu-u.ac.jp

${ }^{1}$ Department of Anesthesiology, Fukuoka University School of Medicine,

7-45-1, Nanakuma, Jonan-ku, Fukuoka 814-0180, Japan

Full list of author information is available at the end of the article
}

(c) The Author(s). 2019 Open Access This article is distributed under the terms of the Creative Commons Attribution 4.0 International License (http://creativecommons.org/licenses/by/4.0/), which permits unrestricted use, distribution, and reproduction in any medium, provided you give appropriate credit to the original author(s) and the source, provide a link to the Creative Commons license, and indicate if changes were made. The Creative Commons Public Domain Dedication waiver (http://creativecommons.org/publicdomain/zero/1.0/) applies to the data made available in this article, unless otherwise stated. 


\section{Background}

Continuous epidural infusion (CEI) of a local anesthetic combined with patient-controlled analgesia (PCA) is an effective postoperative analgesic approach for thoracic surgery [1]. However, CEI has some disadvantages, such as increased local anesthetic consumption and a limited area of anesthetic distribution [2].

Programmed intermittent bolus (PIB) is a technique of epidural anesthesia in which boluses of local anesthetic are automatically injected into the epidural space. This technique increases the analgesic area [3]. Reports have indicated that intermittent epidural bolus administration reduces local anesthetic usage and improves maternal satisfaction in labor analgesia [4-6]. However, the usefulness of PIB in thoracic surgery is unclear.

The purpose of this study was to compare the efficacies of PIB epidural analgesia and CEI in patients undergoing thoracic surgery.

\section{Methods}

This randomized prospective study was approved by the Institutional Review Board (IRB No. 15-9-06) of Fukuoka University Hospital, Fukuoka, Japan, and was registered in the clinical trials database UMIN (ID 000019904) on 24
November 2015. Written informed consent was obtained from all patients.

\section{Patients}

Patients undergoing open lung lobectomy or partial lobectomy at the Fukuoka University Hospital, Fukuoka, Japan between March 2016 and March 2017 were recruited. The exclusion criteria were age $<20$ years and contraindication for epidural anesthesia. Patients were randomly divided into a CEI or PIB group by computer generated randomization using Excel 2013 (Microsoft Inc., Redmond, WA) by KY (Fig. 1).

The study was discontinued when epidural analgesia was ineffective, when the patient's hypotension continued even after the administration of vasopressor, or when motor paralysis appeared owing to epidural analgesia.

\section{Anesthesia}

Under standard monitoring, thoracic epidural anesthesia was performed at Th4-6 in the lateral position. An 18G epidural Tuohy needle (Uniever ${ }^{\bullet}$, Unisis Corp., Saitama, Japan) was used, and the epidural space was identified using the loss-of-resistance technique. A 20G epidural catheter (Uniever, Unisis Corp.) was inserted $5 \mathrm{~cm}$ to the head side. Following a 3-mL test dose of $1 \%$ mepivacaine, the epidural catheter was fixed.

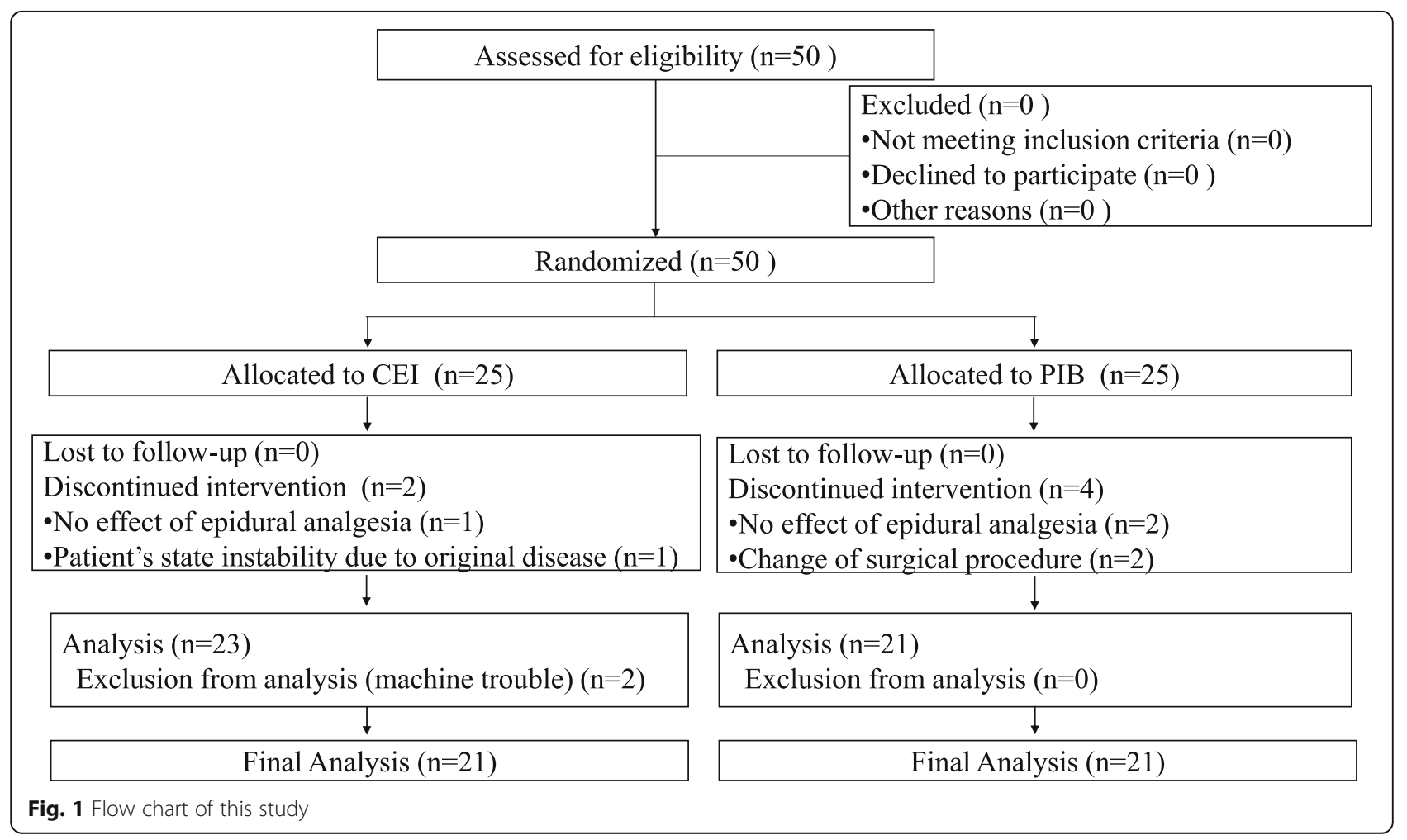


General anesthesia was induced with intravenous fentanyl $(2 \mu \mathrm{g} / \mathrm{kg})$, propofol $(1 \mathrm{mg} / \mathrm{kg})$, and rocuronium $(0.9 \mathrm{mg} / \mathrm{kg})$ and was maintained with sevoflurane $(1.5-2 \%)$ and remifentanil $(0.1-0.2 \mu \mathrm{g} / \mathrm{kg} / \mathrm{min})$. Fentanyl was used intravenously up to $5 \mu \mathrm{g} / \mathrm{kg}$. A local anesthetic via the epidural catheter was not used during the operation.

After surgery, all patients were extubated in the operating room, observed in the post-anesthesia care unit for $30 \mathrm{~min}$ to $1 \mathrm{~h}$, and then transferred to the ward.

\section{PIB group}

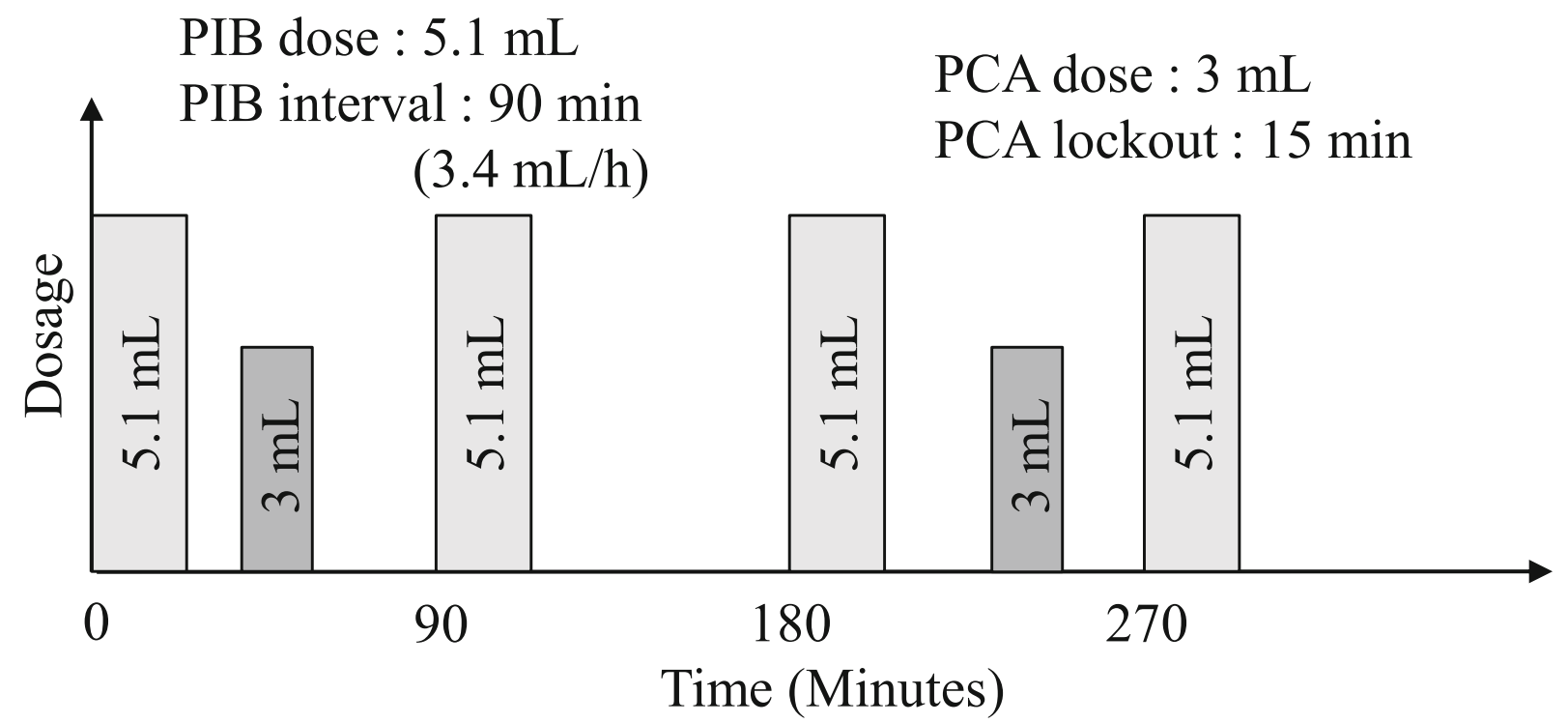

\section{CEI group}

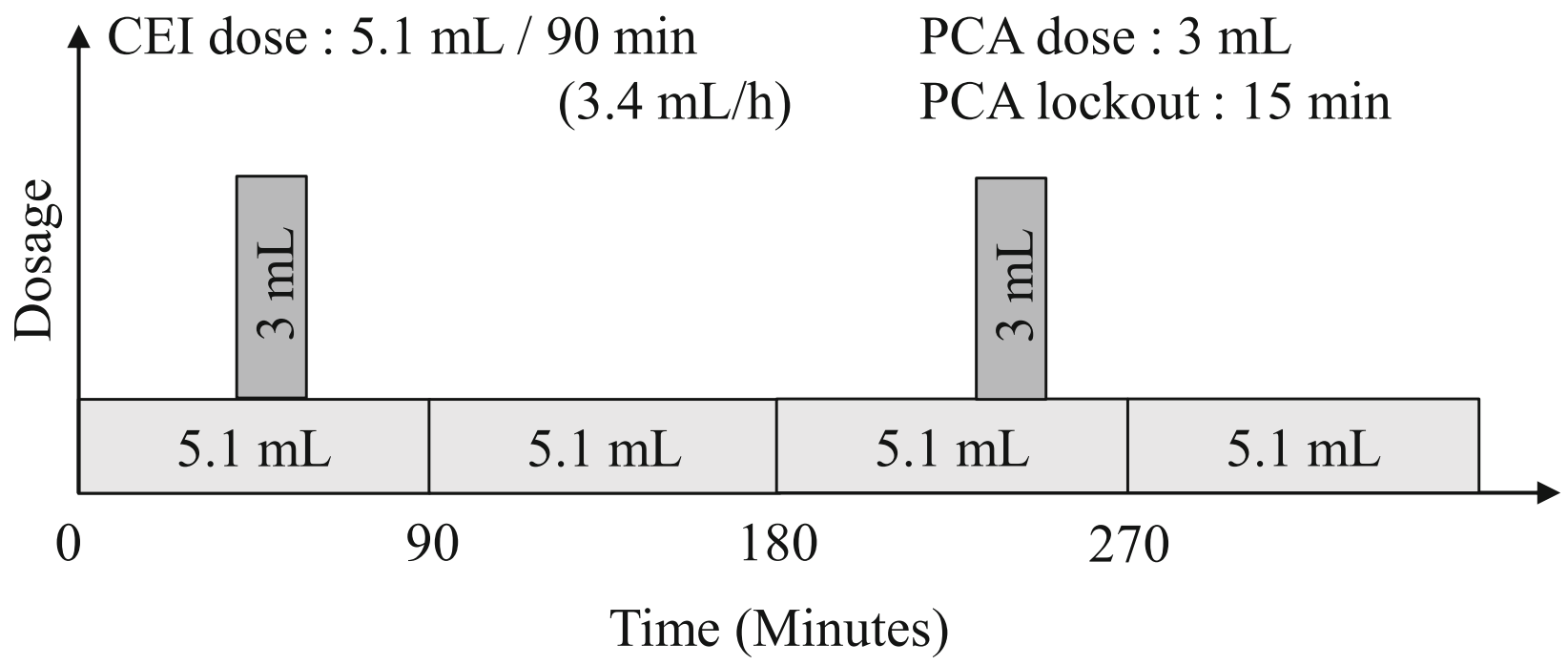

Fig. 2 Study protocol. PIB: programmed intermittent bolus, PCA: patient controlled analgesia, CEl:continuous epidural infusion 
Table 1 Patient characteristics

\begin{tabular}{llll}
\hline & PIB $(n=21)$ mean \pm SD & CEI $(n=21)$ mean \pm SD & $P$-value \\
\hline Age (years) & $63 \pm 2.8$ & $67 \pm 2.6$ & 0.34 \\
BMI $\left(\mathrm{kg} / \mathrm{m}^{2}\right)$ & $24.5 \pm 1.1$ & $22.4 \pm 0.5$ & 0.08 \\
SBP $(\mathrm{mmHg})$ & $122 \pm 2$ & $121 \pm 2$ & 0.72 \\
Use of analgesics during the operation & & & $0.63 \pm 35$ \\
$\quad$ Fentanyl $(\mu \mathrm{g})$ & $171 \pm 35$ & $1.8 \pm 0.2$ & 0.32 \\
$\quad$ Remifentanil (mg) & $1.5 \pm 0.2$ & $263 \pm 18$ & 0.61 \\
Operation time (min) & $249 \pm 20$ & $351 \pm 18$ & 0.56 \\
Anesthesia time (min) & $334 \pm 22$ & & \\
\hline
\end{tabular}

\section{Intervention}

At the end of surgery, a 5-mL initial dose of local anesthetic (ropivacaine $2 \mathrm{mg}$ and fentanyl $2 \mu \mathrm{g}$ in $1 \mathrm{ml}$ ) was administered via the epidural catheter after closure of thoracotomy in both groups.

The study protocol is shown in Fig. 2. In both the PIB and CEI groups, a pump (CADD-Solis ambulatory infusion pump, Smith Medical, St Paul, MN, USA) was used. In the CEI group, patients received continuous infusion of the local anesthetic at a rate of $5.1 \mathrm{~mL} / 90 \mathrm{~min}$ $(3.4 \mathrm{~mL} / \mathrm{h})$. In the PIB group, the pump delivered the local anesthetic at a dose of $5.1 \mathrm{~mL}$ every $90 \mathrm{~min}$. The PCA system was programmed to deliver a 3-mL bolus of the local anesthetic with a lockout interval of $15 \mathrm{~min}$ in both groups.

The primary endpoints were the frequency of PCA and total dose of local anesthetic during $36 \mathrm{~h}$ of postoperative period. The secondary endpoints were pain intensity, frequency of rescue analgesics, including nonsteroidal anti-inflammatory drugs and acetaminophen, adverse events, hypotension, and postoperative nausea and vomiting (PONV). Hypotension was defined as systolic blood pressure (SBP) 20\% less than the baseline value or less than $90 \mathrm{mmHg}$. The onset of adverse reactions and use of rescue analgesics postoperatively were examined. The pain intensity was assessed using a visual analog scale (VAS) during rest, deep breathing, cough, and movement.

\section{Statistical analysis}

Continuous variables are expressed as mean \pm standard deviation or median [lower-upper quartiles]. From the results of preliminary study, total dose of local anesthetics in CEI was $24 \mathrm{ml}$ more than in PIB,

\section{A. Frequency of administrated PCA}

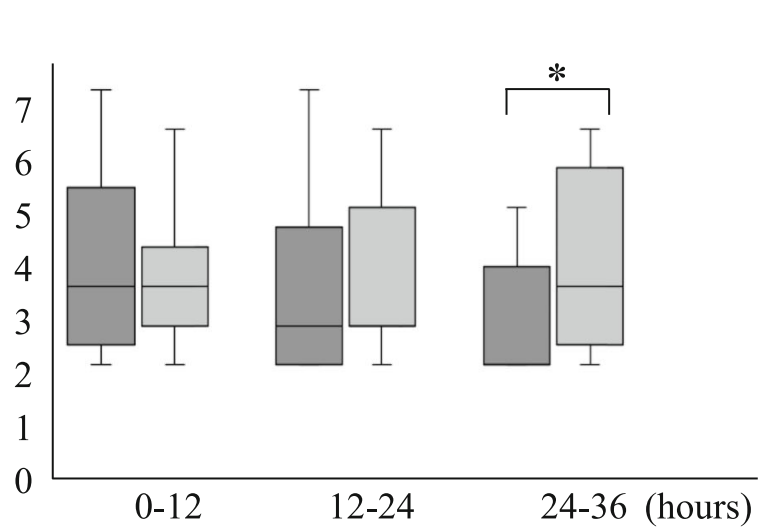

\section{B. Quantity of local anesthetic}

$(\mathrm{mL})$

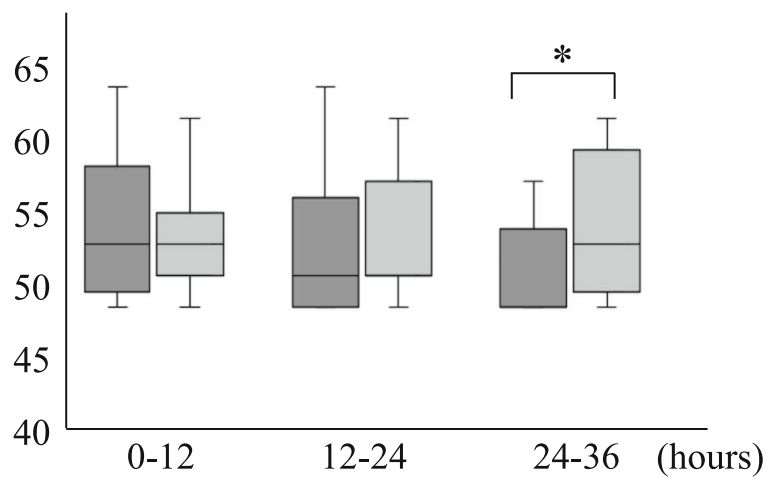

Fig. 3 PCA data after surgery. Data are presented as median [lower-upper quartiles]. The Mann-Whitney $U$ test was used for comparison of categorical variables. ${ }^{*} P<0.05$. PCA: patient-controlled analgesia, PIB: programmed intermittent bolus, CEl: continuous epidural infusion 
Table 2 Use of rescue analgesics postoperatively

\begin{tabular}{|c|c|c|c|}
\hline & $\mathrm{PIB}(n=21)$ & CEI $(n=21)$ & \multirow[t]{2}{*}{$P$-value } \\
\hline & n (\%) & n (\%) & \\
\hline \multicolumn{4}{|l|}{ Use of analgesics } \\
\hline Loxoprofen & 20(95.2) & 19(85.6) & 0.55 \\
\hline Acetaminophen & $1(4.7)$ & $3(14.3)$ & 0.61 \\
\hline Celecoxib & $1(4.7)$ & $2(9.5)$ & 0.55 \\
\hline Tramadol & $2(9.5)$ & $1(4.7)$ & 0.55 \\
\hline
\end{tabular}

and SD of CEI was 32. Based on these results, we estimated that the following: $\mathrm{SD}=32, \Delta=0.78, \alpha=0.05$, and beta $=0.2$. The required number of cases was estimated to be 21 for each group. We considered a 10-20\% dropout rate; therefore, 50 patients were enrolled. Differences between groups were examined for statistical significance by using student's $t$-test after logarithmic transformation. Student's $t$-test, the chi-square test, and the Mann-Whitney $U$ test were used for statistical analyses. A $P$-value $<0.05$ was considered statistically significant.

\section{Results}

Fifty patients who underwent open lung lobectomy or partial lobectomy were randomly divided into the CEI group $(n=25)$ and PIB group $(n=25)$. In the CEI group, 2 patients were excluded because of ineffectiveness of epidural analgesia and instability in the patient's state due to the original disease. In the remaining 23 patients of the CEI group, additional 2 patients were excluded from the analysis because of machine trouble; finally, 21 patients were included in the analysis. In PIB group, 4 patients were excluded (2 patients owing to ineffectiveness of epidural analgesia and 2 patients owing to change in surgical procedure), and 21 patients were finally included in the analysis (Fig. 1). Patient characteristics are shown in Table 1.

The mean number of PCA administrations and total amount of local anesthetic were not significantly different between the two study groups up to $24 \mathrm{~h}$ after surgery. However, the mean number of PCA administrations was significantly lower in the PIB group than in the CEI group at 24-36 h after surgery (median [lowerupper quartiles]: 0 [0-2.5] vs. 2 [0.5-5], $P=0.018)$ and total amount of local anesthetic was also significantly lower in the PIB group than in the CEI group at 24-36 h after surgery (median [lower-upper quartiles]: 41 [4148.5 ] vs. 47 [43-56] $\mathrm{mL}, P=0.035$ ) (Fig. 3). The use of rescue analgesics was not significantly different between the two study groups (Table 2). The VAS scores during

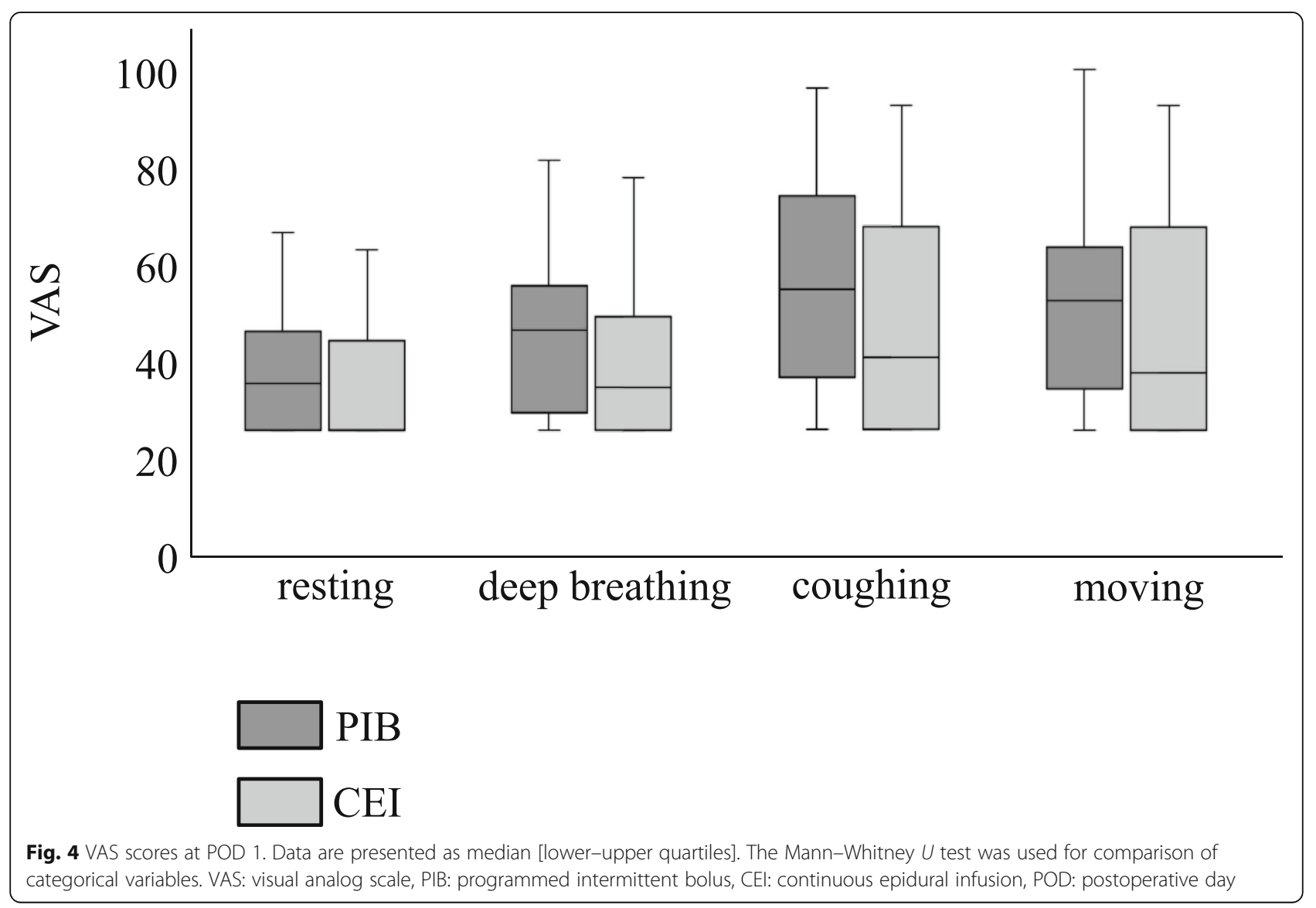


Table 3 Adverse events

\begin{tabular}{llll}
\hline & PIB $(n=21)$ & CEI $(n=21)$ & $P$-value \\
& $\mathrm{n}(\%)$ & $\mathrm{n}(\%)$ & \\
\hline Adverse effects & & & \\
Nausea, vomiting & $8(38.1)$ & $5(23.8)$ & 0.32 \\
Urinary retention & $2(9.5)$ & $3(14.3)$ & 0.63 \\
Feeling dizzy on standing up & $4(19.0)$ & $1(4.7)$ & 0.15 \\
\hline
\end{tabular}

resting, deep breathing, coughing, and moving after the surgery were also not significantly different between the two study groups (Fig. 4).

The frequencies of adverse events, such as nausea, vomiting, and dizziness on standing up, were not significantly different between the two study groups (Table 3). The frequency of hypotension was greater in the PIB group than in the CEI group at $0-12 \mathrm{~h}$ and $12-24 \mathrm{~h}$ postoperatively $(3.3 \%$ vs. $0.5 \%, P=0.018$ and $7.9 \%$ vs. $0 \%$, $P=0.017$, respectively) (Table 4$)$.

\section{Discussion}

Our results showed that PIB has an analgesic effect comparable with that of $\mathrm{CEI}$ and reduces the required amount of local anesthetic on the first day after thoracotomy. However, adverse events, such as hypotension, need attention.

To compensate for the limitations of CEI, such as a restricted area of analgesic effect, the technique of intermittent bolus infusion of epidural analgesics has been developed. The advantage of PIB is mainly in the maintenance of labor analgesia [5, 7]. Its use has been recently demonstrated in total knee arthroplasty and major abdominal and gynecological surgery, and its utility has been shown [8-10]. However, to our knowledge, this is the first randomized study to show the advantage of PIB in thoracotomy.

The reduction in the total amount of local anesthetic with intermittent bolus infusion compared with continuous infusion is consistent with the findings in labor analgesia reports and postoperative reports. In major

Table 4 Frequency of hypotension events after surgery

\begin{tabular}{cccc}
\hline & $\mathrm{PIB}(n=21)$ & $\mathrm{CEI}(n=21)$ & P-value \\
& $\mathrm{n}(\%)$ & $\mathrm{n}(\%)$ & \\
\hline 20\% less than baseline SBP & & \\
0-12 h & $7(3.3)$ & $1(0.5)$ & 0.018 \\
$12-24 \mathrm{~h}$ & $6(9.5)$ & $3(4.7)$ & 0.26 \\
24-36 h & $6(9.5)$ & $4(6.3)$ & 0.47 \\
SBP less than $90 \mathrm{mmHg}$ & & \\
0-12 h & $10(4.8)$ & $4(1.9)$ & 0.0495 \\
12-24 h & $5(7.9)$ & $0(0.0)$ & 0.017 \\
24-36 h & $4(6.3)$ & $1(1.6)$ & 0.15 \\
\hline
\end{tabular}

abdominal and gynecological surgery, the beneficial effect of PIB is noted on the first postoperative day and not on the day of the operation [9]. Sequential epidural bolus infusion provides superior epidural block compared with CEI [2].

Compared with bolus infusion, hemodynamic stability with CEI without bolus administration is superior; the incidence of hypotension reduced by $67 \%$ without using bolus infusion compared with that using bolus infusion [11]. However, PIB studies for postsurgical analgesia indicated no adverse effects [8-10]. With regard to the incidence of hypotension, the difference between our results and those of previous reports might be associated with differences in the site of epidural anesthesia and dose of local anesthetic. Hypotension occurred but was not significant in both groups, and there was a need for noradrenalin when epidural anesthesia involved puncture at Th8-10 [9]. On the other hand, when epidural anesthesia involved puncture at Th10-12 in open gynecological surgery [8] or L3-5 in total knee arthroplasty, [8] there was no hypotension requiring intervention. The bolus dose was $6 \mathrm{~mL}$ every hour in the major surgical study that reported hypotension, [9] and among studies that did not report hypotension, the doses were $4 \mathrm{~mL}$ every hour for open gynecological surgery [10] and $3 \mathrm{~mL}$ every hour for total knee arthroplasty [8]. We used a bolus of $5.1 \mathrm{~mL}$ every $90 \mathrm{~min}$ (3.4 mL every hour). Therefore, when PIB and CEI are used for a higher level of thoracic epidural anesthesia, attention should be paid to the bolus dose to avoid hypotension.

The present study has limitations. First, this is not double blinded study. Second, in this study, the dose and concentration of local anesthetic was single, and the total dose of local anesthetic and counts of PCA were less than that of preliminary studies. We need to reexamine the small dose and concentration of local anesthetics in future studies.

\section{Conclusions}

PIB can reduce local anesthetic consumption in thoracic surgery. However, it might result in adverse events, such as hypotension.

\section{Abbreviations}

BMl: Body mass index; CEl: Continuous epidural infusion; PCA: Patient-controlled analgesia; PIB: Programmed intermittent bolus; PONV: Postoperative nausea and vomiting; SBP: Systolic blood pressure; UMIN: University hospital medical information network; VAS: Visual analog scale

\section{Acknowledgements}

The authors would like to thank Enago (https://www.enago.jp/) for the English language review.

Authors' contributions

$\mathrm{MH}, \mathrm{KY}$ : conceptualized and designed the study and wrote the manuscript; KS: analyzed data; and EN, SS: collected the data. All authors read and approved the manuscript. 
Funding

None of the authors received any additional funding in regard to this paper or related to this paper's data.

\section{Availability of data and materials}

The datasets generated and/or analyzed during the current study will be available from the corresponding author on reasonable request.

\section{Ethics approval and consent to participate}

This randomized prospective study was approved by the Institutional Review Board (IRB No. 15-9-06) of Fukuoka University Hospital, Fukuoka, Japan, and was registered in the clinical trials database UMIN (ID 000019904) on 24 November 2015. Written informed consent was obtained from all patients.

\section{Consent for publication}

Not applicable.

\section{Competing interests}

The authors declare that they have no competing interests.

\section{Author details}

'Department of Anesthesiology, Fukuoka University School of Medicine, 7-45-1, Nanakuma, Jonan-ku, Fukuoka 814-0180, Japan. ${ }^{2}$ Operation rooms, Fukuoka University Hospital, Fukuoka, Japan.

Received: 3 March 2019 Accepted: 5 June 2019

Published online: 15 June 2019

\section{References}

1. Saeki H, Ishimura H, Higashi H, Kitagawa D, Tanaka J, Maruyama R, et al. Postoperative management using intensive patient-controlled epidural analgesia and early rehabilitation after an esophagectomy. Surg Today. 2009:39:476-80

2. Ueda K, Ueda W, Manabe M. A comparative study of sequential epidural bolus technique and continuous epidural infusion. Anesthesiology. 2005; 103:126-9.

3. Patkar CS, Vora K, Patel H, Shah V, Modi MP, Parikh G. A comparison of continuous and intermittent bolus administration of $0.1 \%$ ropivacaine with $0.0002 \%$ fentanyl for epidural labor analgesia. J Anaesthesiol Clin Pharmacol. 2015;31:234-8

4. George RB, Allen TK, Habib AS. Intermittent epidural bolus compared with continuous epidural infusions for labor analgesia: a systematic review and meta-analysis. Anesth Analg. 2013;116:133-44.

5. Carvalho B, George RB, Cobb B, McKenzie C, Riley ET. Implementation of programmed intermittent epidural bolus for the maintenance of labor analgesia. Anesth Analg. 2016;123:965-71.

6. Sng BL, Zeng Y, de Souza NNA, Leong WL, Oh TT, Siddiqui FJ, et al. Automated mandatory bolus versus basal infusion for maintenance of epidural analgesia in labour. Cochrane Database Syst Rev. 2018:5:CD011344.

7. Onuoha OC. Epidural analgesia for labor: continuous infusion versus programmed intermittent bolus. Anesthesiol Clin. 2017;35:1-14.

8. Kang S, Jeon S, Choe JH, Bang SR, Lee KH. Comparison of analgesic effects of programmed intermittent epidural bolus and continuous epidural infusion after total knee arthroplasty. Korean J Anesthesiol. 2013;65:S130-1.

9. Wiesmann $T$, Hoff $L$, Prien $L$, Torossian A, Eberhart L, Wulf $H$, et al. Programmed intermittent epidural bolus versus continuous epidural infusion for postoperative analgesia after major abdominal and gynecological cancer surgery: a randomized, triple-blinded clinical trial. BMC Anesthesiol. 2018;18:154

10. Satomi S, Kakuta N, Murakami C, Sakai Y, Tanaka K, Tsutsumi YM. The efficacy of programmed intermittent epidural bolus for postoperative analgesia after open gynecological surgery: a randomized double-blinded study. Biomed Res Int. 2018. https://doi.org/10.1155/2018/6297247.

11. Gerhardt MA, Gunka VB, Miller RJ. Hemodynamic stability during labor and delivery with continuous epidural infusion. J Am Osteopath Assoc. 2006;106: 692-8.

\section{Publisher's Note}

Springer Nature remains neutral with regard to jurisdictional claims in published maps and institutional affiliations.

Ready to submit your research? Choose BMC and benefit from:

- fast, convenient online submission

- thorough peer review by experienced researchers in your field

- rapid publication on acceptance

- support for research data, including large and complex data types

- gold Open Access which fosters wider collaboration and increased citations

- maximum visibility for your research: over $100 \mathrm{M}$ website views per year

At BMC, research is always in progress.

Learn more biomedcentral.com/submissions 